\title{
Temperature Dependence of the Primary Species Yields of Liquid Water Radiolysis by 0.8-MeV Fast Neutrons
}

\author{
S.L. Butarbutar ${ }^{1,2^{*}}$, G.R. Sunaryo ${ }^{1}$, J. Meesungnoen ${ }^{2}$ and J.P. Jay-Gerin ${ }^{2}$ \\ ${ }^{I}$ Center for Nuclear Reactor Technology and Safety, National Nuclear Energy Agency \\ Puspiptek Area, Serpong, Tangerang Selatan 15314, Indonesia \\ ${ }^{2}$ Département de médecine nucléaire et de radiobiologie, Faculté de médecine et des sciences de la santé, \\ Université de Sherbrooke 3001, $12^{e}$ Avenue Nord, Sherbrooke (Québec) J1H 5N4, Canada
}

\section{ARTICLE INFO}

Article history:

Received 04 October 2014

Received in revised form 23 March 2016

Accepted 23 March 2016

\section{Keywords:}

Fast neutrons

Radiolysis

High temperature

Yields ( $G$-values)

\begin{abstract}
A B S T R A C T
The yields of species such as $\mathrm{e}_{\mathrm{aq}}^{-}, \mathrm{H}^{\bullet},{ }^{\circ} \mathrm{OH}, \mathrm{H}_{2}$ and $\mathrm{H}_{2} \mathrm{O}_{2}$, formed from the radiolysis of neutral liquid water by the incidence of $0.8-\mathrm{MeV}$ neutrons at temperatures between 25 and $350^{\circ} \mathrm{C}$, were calculated by using Monte Carlo simulations. The slowing down of these neutrons through elastic scattering produced recoil protons elastically of $\sim 0.5057,0.186$, and $0.0684 \mathrm{MeV}$ which had linear energy transfers (LETs) of $\sim 40,67$ and $76 \mathrm{keV} / \mu \mathrm{m}$, respectively, at $25^{\circ} \mathrm{C}$. The effects of neutron radiation can be predicted based on the contribution of those first three recoil protons by neglecting the radiation effects due to oxygen ion recoils. Then, the fast neutron yields could be estimated by summing the yields of contributing protons after corresponding weightings were used according to their energy. In this work, yields were calculated at $10^{-7}$ and $10^{-6} \mathrm{~s}$ after incidence of neutron radiation in water at the aforementioned temperature range. Overall, there is a reasonably good agreement between our calculated and existing experimental $G$-values for the entire temperature range. However, we proposed an hypothesis that the not very significant difference between experimental data and our calculated data is due to the different measuring time used in obtaining the experimental data as compared to the ones used in our calculation. Our computed yields for $0.8-\mathrm{MeV}$ fast neutron radiation show an essentially similar temperature dependences over the range of temperature studied with 2-MeV fast neutron and low-LET radiation, but with lower values for yields of free radicals and higher values for molecular yields.
\end{abstract}

(C) 2016 Atom Indonesia. All rights reserved

\section{INTRODUCTION}

In water-cooled nuclear power reactors, such as the Pressurized Water Reactor (PWR), water is used both as a moderator and as a heat transport medium. It circulates around the reactor core at a normal operating temperature of $\sim 250-310^{\circ} \mathrm{C}$ and is heavily irradiated by a mixture of radiation fields such as gamma radiation, fast electrons, fast neutrons and so forth. It interacts directly with those radiations fields that can cause radiolysis of water (decomposition of water by nuclear radiation) which generate free radicals (such as $\mathrm{e}_{\text {aq }}^{-},{ }^{\circ} \mathrm{OH}$, and $\mathrm{H}^{*}$ )

*Corresponding author.

E-mail address: sofia@batan.go.id

DOI: http://dx.doi.org/10.17146/aij.2016.473 and molecular products (such as $\mathrm{H}_{2}$ and $\mathrm{H}_{2} \mathrm{O}_{2}$ ). Oxydizing species resulting from water radiolysis such as ${ }^{\circ} \mathrm{OH}, \mathrm{H}_{2} \mathrm{O}_{2}$, and $\mathrm{O}_{2}$ (secondary product of radiolysis) can interact with reactor components material and promote corrosion, hydriding, and cracking [1-4]. For these reasons, the water chemistry in a water-cooled nuclear power plant has to be controlled. Furthermore, the water chemistry control requires an understanding of the effects of radiolysis of water that may degrade reactor component material.

At least, there are two important parameters which need to be determined, namely the yields ( $G$-values) of the species (the number of molecules formed or destroyed per $100 \mathrm{eV}$ absorbed energy, expressed in the units of molecules $/ 100 \mathrm{eV}$ ) and the 
rate constants of all the reactions between radiolytic species. Direct measurement of the chemistry in and around reactor core is difficult or impossible to perform due to the high temperature, high pressure, and intense radiation fields. For these reasons, a computer simulation is a better way to predict the mechanism of radiolysis of cooling water and the effect of its products on reactor materials.

In this work, we used Monte Carlo simulations to reproduce $G$-values for $\mathrm{e}^{-}{ }_{\mathrm{aq}},{ }^{\circ} \mathrm{OH}, \mathrm{H}^{\circ}$, $\mathrm{H}_{2}$, and $\mathrm{H}_{2} \mathrm{O}_{2}$ generated from pure liquid water radiolysis by $0.8-\mathrm{MeV}$ fast neutrons at the temperature range of $25-350^{\circ} \mathrm{C}$. The reason we only considered $0.8-\mathrm{MeV}$ fast neutrons in this present work is that this energy is largely known as the average energy of the neutron source reactor YAYOI in Japan (where 95\% of the radiation energy of the radiation energy came from fast neutrons [5]). Peculiarly, there are limited data of the fast neutron $G$-values in the literature as compared to the gamma ones, especially at high temperatures. However, Sunaryo et al. [6] have simulated this energy at the temperature range of $25-325^{\circ} \mathrm{C}$. Furthermore, there is much updated information which has been incorporated in the code developed by J.-P. Jay-Gerin's group in Université de Sherbrooke, Québec, Canada as follows. First, the information of the rate constants of the reactions between species for up to $350^{\circ} \mathrm{C}$ are now available from the report compiled by Elliot and Bartels [7] and have been used in several works [8-10]. Second, the rate constants of two reactions that remain debatable, namely the ones for the selfrecombination of hydrated electron $\left(\mathrm{e}_{\mathrm{aq}}^{-}\right.$) above $\sim 150^{\circ} \mathrm{C}[11,12]$ and for the oxidation reaction of water by hydrogen radical atoms above $\sim 200^{\circ} \mathrm{C}$ $[13,14]$, are incorporated in the simulation.

In this work, principally the effects of $0.8-\mathrm{MeV}$ fast neutron were predicted by calculating the yields of the first three recoil protons and by neglecting the radiation effects due to oxygen ion recoils $[15,16]$. We finally chose the contributions of the first three recoil protons after some tests have been conducted. In the previous work, we found that there is no significant difference between taking the first three and the first four recoil protons. The less the energy of recoil protons released from neutron collision with water is, the less their contribution to the final yield is, since appropriate weighting factors are applied according to their energies to calculate yields. Therefore, the fourth, the fifth and so forth of recoil protons will not make any difference to our computed yields. The results were tested against the available experimental and calculated data.

\section{THEORY}

The interaction of neutron with material strongly depends on its kinetic energy. For fast neutrons with kinetic energies ranging from $\sim 0.5$ to $10 \mathrm{MeV}$, the possible interactions of neutron with material mostly consist of elastic collisions. During this interaction, the total kinetic energy before and after collision remains the same. Thus, this interaction generates the low-kinetic-energy particles in the reactor. In case of water, as neutrons collide with nuclei, fast neutrons are "moderated" mainly by both hydrogen (proton) and oxygen nuclei. For water, the spectrum of recoil proton and oxygen energies can be determined and thus can be used to determine the chemical yields for the various species formed from the fast neutron-water interaction.

It is well-known that fast neutrons deposit their energies in a relatively dense track as compared to $\gamma$-radiation. The quantity that most significantly characterizes neutron transmission through matter is the mean-free-path (i.e., the mean distance between two successive neutron interactions). For example, the mean free path of a $0.8-\mathrm{MeV}$ incident neutron in water at $25^{\circ} \mathrm{C}$ is $\sim 3 \mathrm{~cm}$, while the recoil proton and oxygen ion maximum ranges at this energy are in $\mu \mathrm{m}$ order $[15,16]$. Thus, it can be inferred that proton and oxygen ion recoils in $\mathrm{H}_{2} \mathrm{O}$ are widely separated from one another along the path of the neutron and their ranges are much less than the average separation between two successive neutron interactions, so there is no overlap between one and another.

For energies less than $\sim 10 \mathrm{MeV}$, it is estimated that about $88 \%$ of the neutron energy is absorbed by recoil protons and the rest is absorbed by oxygen nuclei. From those numbers, it is obvious that oxygen ion recoils give a relatively small contribution to the radiolysis, in addition to the fact that the water molecule $\left(\mathrm{H}_{2} \mathrm{O}\right)$ consists of two hydrogen atoms but only one oxygen atom; for those reasons, it is reasonable to simply neglect the radiation effects due to these recoil oxygen ions, or in other words, assume that neutrons are stopped by protons only $[15,16]$.

As in the previous work, the energy of a recoil proton can be calculated using the following equation $[15,16]$ :

$$
\ln \left(\frac{E_{0}}{E_{n}}\right)=n\left[1+\frac{(A-1)^{2}}{2 A} \ln \left(\frac{A-1}{A+1}\right)\right]
$$

where:

- $A=$ the mass number of the struck nucleus ( $A=1$ for collisions with protons) 
- $E_{0}=$ the initial neutron kinetic energy

- $E_{n}=$ the average energy of the neutron after $n$ collisions

Note that when $A=1$, Eq. (1) is not defined; however, the limit is valid. We used Monte Carlo track chemistry simulations of the radiolysis of water to estimate the effects of $0.8-\mathrm{MeV}$ neutrons on the chemical yields of the various radiolytic species by using three recoil protons. By using Eq. (1) we found that the first three recoil protons have energies of $0.5057,0.186$, and $0.0684 \mathrm{MeV}$, respectively.

Then, the final neutron yield can be expressed as $[15,16]$ :

$$
G(\mathrm{X})=\frac{\sum_{\mathrm{i}=1}^{3}\left(G(\mathrm{X}){ }_{p_{\mathrm{i}}} E_{p_{\mathrm{i}}}\right)}{E_{\mathrm{T}}},
$$

where: $G(\mathrm{X})_{p \mathrm{i}}$ is the free radical or molecular yield associated with the recoil proton $p \mathrm{i}(\mathrm{i}=1$ to 3$)$; while $E_{p \mathrm{i}}=\left(E_{\mathrm{i}}-E_{\mathrm{i}-1}\right)$ is the energy imparted to the $i^{\text {th }}$ recoil proton (thus, the energies imparted to the three recoil protons are $E_{p 1}, E_{p 2}$, and $\left.E_{p 3}\right)$; and:

$$
E_{\mathrm{T}}=\sum_{\mathrm{i}=1}^{3} E_{p_{\mathrm{i}}}
$$

is the sum of all recoil proton energies.

\section{METHODOLOGY}

The analysis of the complex series of steps involved in the radiolysis of liquid water by colliding protons of various initial energies were performed through Monte Carlo simulations using the IONLYS-IRT code. A detailed description of the code at both ambient and elevated temperatures has previously been given elsewhere [17-19]. Briefly, in the IONLYS code, the early physical and physicochemical stages of the radiation event were modeled up to $\sim 10^{-12} \mathrm{~s}$. Then, the output of this model is used as the input for the IRT code that covers the nonhomogeneous chemical stage at time scale from $\sim 10^{-12} \mathrm{~s}$ to $\sim 10^{-7}-10^{-6} \mathrm{~s}$ at $25^{\circ} \mathrm{C}$. The detailed elucidation of IONLYS code and the independent reaction times (IRT) method [19-21] had been published in the previous manuscripts. In this present work, information such as rate constants, diffusion coefficients, reaction mechanisms, and $G$-values for the temperature up to $350^{\circ} \mathrm{C}$ were obtained from the report of Elliot and Bartels [7]. The rate constant of the two reactions that concern us in this work, namely the selfreaction of two hydrated electrons and reaction between water molecules and hydrogen atoms, were incorporated using the information available in [7], consecutively.

In addition, the density of the pressurized water used in all simulations decreased from 1 $\mathrm{g} / \mathrm{cm}^{3}$ at $25^{\circ} \mathrm{C}$ to $0.575 \mathrm{~g} / \mathrm{cm}^{3}(16.5 \mathrm{MPa})$ at $350^{\circ} \mathrm{C}[19]$.

Finally, in order to reproduce the effects of the $0.8-\mathrm{MeV}$ neutrons, short $(\sim 15-150 \mu \mathrm{m})$ track segments of each of the first three generated recoil protons were simulated, while the number of proton histories used were usually $~ 10-150$ depending on the proton energy. The total neutron yield for each radiolytic products were then calculated by summing the corresponding weighted $G$-values for each recoil proton according to Eq. (2).

\section{RESULTS AND DISCUSSION}

The computed yields of radiolytic species such as $\mathrm{e}^{-}{ }_{\text {aq }}, \mathrm{OH}, \mathrm{H}^{\circ}, \mathrm{H}_{2} \mathrm{O}_{2}$, and $\mathrm{H}_{2}$ in liquid water formed by $0.8-\mathrm{MeV}$ neutrons as a function of temperature in the range of $25-350^{\circ} \mathrm{C}$ are shown in Fig. 1. In this work, $G$-values were taken at two different times, specifically $10^{-7}$ and $10^{-6} \mathrm{~s}$ after the passage of ionizing radiation through water, at the entire temperature range previously mentioned (solid and dashed red lines in Fig. 1, respectively). The reason we took yields at those times is that the scavenging time (product of the reciprocal of scavenger concentration and its rate constant with one of the radiolytic species) normally used in scavenging experiments to measure the yields is in the range of $\sim 10^{-7}-10^{-5} \mathrm{~s}$. Overall, there is a reasonably good agreement between the simulated and existing experimental $G$-values for the entire temperature range. However, it is obvious that there is only limited information in the literature for fast neutron radiolysis, while a large body of data relevant to the $\gamma$-ray and fast electron radiolysis is readily available for temperatures ranging from room temperature up to high temperatures. In fact, to the best of our knowledge, until now, the data from Sunaryo et al. [5] is the only data available in the literature (using neutron source reactor YAYOI, whose average energy of neutron is about $0.8 \mathrm{MeV}$ ) with which we can compare our results on the temperature dependence of various primary species yields for liquid water irradiated with fast neutrons.

From Fig. 1(a), it is seen that at temperatures below $150^{\circ} \mathrm{C}$, there is a good agreement between our computed yields and experimental values. However, at higher temperature, our yield slightly decreases as a function of temperature, while experimental data show an increase. 
This discrepancy can be readily explained as we incorporated in our calculation the drop in the rate constant of self-reaction of $\mathrm{e}^{-}$aq (reaction 5 (R5) in Table 1) observed at $\sim 150^{\circ} \mathrm{C}$ in alkaline water to near-neutral water. In other side, the decay of $\mathrm{e}^{-}$aq is mainly due to the spur reactions of $\mathrm{R} 1$ and $\mathrm{R} 2$ (the reactions contribute to the formation and decay of the primary species; data is not shown here), in order of decreasing importance. As a complement, however, Table 1 consist only of reactions that contribute to the formation and decomposition of ${ }^{\circ} \mathrm{OH}$. In fact, there are more than 60 reactions included in the IRT code that occur in the nonhomogeneous chemical stage involving the radiolytic species. As suggested by Fig. 1(a), our calculated data show that the decay of $\mathrm{e}^{-}$aq take place faster at high temperatures and at later time after the passage of the ionizing radiation. For ${ }^{\circ} \mathrm{OH}$, as the main oxidizing radical generated in this $0.8-\mathrm{MeV}$ fast neutron radiolysis, the reactions which contribute to the formation of this radical are (in order of decreasing importance) R6 and R4, whereas its decay is dominated by reactions $\mathrm{R} 2, \mathrm{R} 7$, and $\mathrm{R} 9$.

The $\mathrm{H}^{\cdot}$ radical atom is one of the minor species in water radiolysis. It is relatively small, but is important for fundamental considerations. The production of $\mathrm{H}^{*}$ atom is dominated by the rapid convertion of $\mathrm{e}_{\text {aq }}^{-}$through reaction $\mathrm{R} 1$. There is a very good agreement between our computed yields and experimental data at room temperature. At higher temperatures, deviations between experimental data and calculated data can be readily explained as follows. It is proposed that the experimental data could have been obtained at different time as compared to our calculation time.

Table 1. Main chemical reactions that contribute to the formation and decomposition of ${ }^{\bullet} \mathrm{OH}$ used in simulations.

\begin{tabular}{cl}
\hline Reaction & \multicolumn{1}{c}{ Symbol } \\
\hline R1 & $\mathrm{e}^{-}{ }_{\text {aq }}+\mathrm{H}^{+} \rightarrow \mathrm{H}^{\cdot}$ \\
R2 & $\mathrm{e}^{-}{ }_{\text {aq }}+{ }^{\circ} \mathrm{OH} \rightarrow \mathrm{OH}^{-}$ \\
R3 & $\mathrm{e}_{\text {aq }}^{-}+\mathrm{H}^{\cdot}\left(+\mathrm{H}_{2} \mathrm{O}\right) \rightarrow \mathrm{H}_{2}+\mathrm{OH}^{-}$ \\
R4 & $\mathrm{e}^{-}{ }_{\text {aq }}+\mathrm{H}_{2} \mathrm{O}_{2} \rightarrow{ }^{\cdot} \mathrm{OH}+\mathrm{OH}^{-}$ \\
R5 & $\mathrm{e}_{\text {aq }}^{-}+\mathrm{e}^{-}{ }_{\text {aq }}\left(+2 \mathrm{H}_{2} \mathrm{O}\right) \rightarrow \mathrm{H}_{2}+2 \mathrm{OH}^{-}$ \\
R6 & $\mathrm{H}^{\cdot}+\mathrm{H}_{2} \mathrm{O} \rightarrow{ }^{\circ} \mathrm{OH}+\mathrm{H}_{2}$ \\
R7 & $\mathrm{H}^{\cdot}+{ }^{\circ} \mathrm{OH} \rightarrow \mathrm{H}_{2} \mathrm{O}$ \\
R8 & $\mathrm{H}^{\cdot}+\mathrm{H}^{\cdot} \rightarrow \mathrm{H}_{2}$ \\
R9 & ${ }^{\circ} \mathrm{OH}+{ }^{\circ} \mathrm{OH} \rightarrow \mathrm{H}_{2} \mathrm{O}_{2}$ \\
R10 & $\mathrm{H}^{+}+\mathrm{HO}_{2}^{-} \rightarrow \mathrm{H}_{2} \mathrm{O}_{2}$ \\
\hline
\end{tabular}

There is a high degree of difference between calculated and measured data of $\mathrm{H}_{2} \mathrm{O}_{2}$ yield, as seen in Fig. 1(d), due to the difficulty in measuring the yield since it is decomposed thermally at high temperatures starting from $\sim 100^{\circ} \mathrm{C} . \mathrm{H}_{2} \mathrm{O}_{2}$ formed in the radiolysis of water is found to be the main corrosion product and is involved in the oxidation damage of most alloys. It has long been known that the main precursor of $\mathrm{H}_{2} \mathrm{O}_{2}$ is the self-reaction of ${ }^{\circ} \mathrm{OH}$ through reaction $\mathrm{R} 9$, while the decay is given dominantly by reaction $\mathrm{R} 4$.

As shown in Fig. 1(e), our simulated $G\left(\mathrm{H}_{2}\right)$ obtained at $10^{-6} \mathrm{~s}$ show a large increase with temperature, particularly above $\sim 200-300^{\circ} \mathrm{C}$. This phenomenon can also be seen in the increase of $G\left({ }^{\circ} \mathrm{OH}\right)$ and $G\left(\mathrm{H}_{2}\right)$ and the corresponding decrease of $G\left(\mathrm{H}^{*}\right)$ with temperature, as have been discussed in the previous work [15]. The three main processes which result in signifant production of $\mathrm{H}_{2}$ are reaction R6, R3 and R5. However, a controversy currently exists in the literature regarding the rate constant of R6, including estimates of $10^{4}$ [15] (value used in the present calculations), $2.2 \times 10^{3}$ [7], and $1.75 \times 10^{4}$ [14] $M^{-1} \mathrm{~s}^{-1}$ at $300^{\circ} \mathrm{C}$, depending on the authors. To our knowledge so far, no clear conclusion has been obtained yet on the rate constant of this reaction and as to the real contribution of this potentially important reaction in the radiolysis of water at elevated temperatures.

For the sake of comparison, our computed results were compared with known data for lowLET radiations ( $\gamma$-rays from ${ }^{60} \mathrm{Co}$ or fast electrons) and with the computed yields obtained from the radiolysis of neutral liquid water by $2-\mathrm{MeV}$ monoenergetic neutrons, as a mimic of a fissionneutron flux in a reactor as well. Our computed yields for $0.8-\mathrm{MeV}$ fast neutron radiation show essentially similar temperature dependences over the range of temperature studied with $2-\mathrm{MeV}$ fast neutrons and low-LET radiations, but with lower values for yields of free radicals and higher values for molecular yields. This general trend is a result of the high-LET character of fast neutrons. As the LET of the radiation increases (the less the energy), many radical interactions occurred therefore the higher the yield of molecular products. Also, we compared our present work with the work of Sunaryo et al. [6], but at this time we used the information available at [7] in the code. Indeed, Sunaryo et al. did not take into account the reaction rate constant of the two reactions discussed previously. It can be seen clearly in Fig. 1(a), where there is no abrupt drop around $\sim 150^{\circ} \mathrm{C}$, and also at their result in Fig. 1(e), as it is known that the self-reaction of hydrated electrons is one source of hydrogen molecular formation. Hence, there is a significant difference between our present work and those works due to the contributions of the rate constants of the two reactions mentioned earlier. 


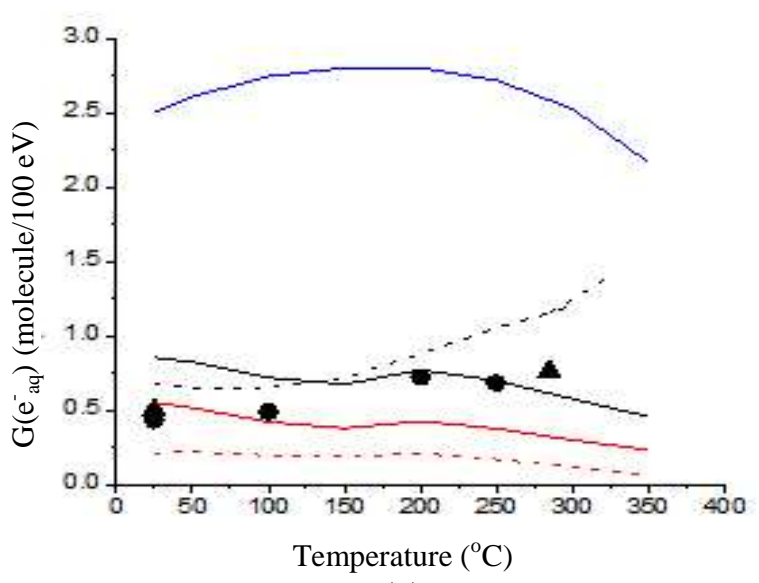

(a)

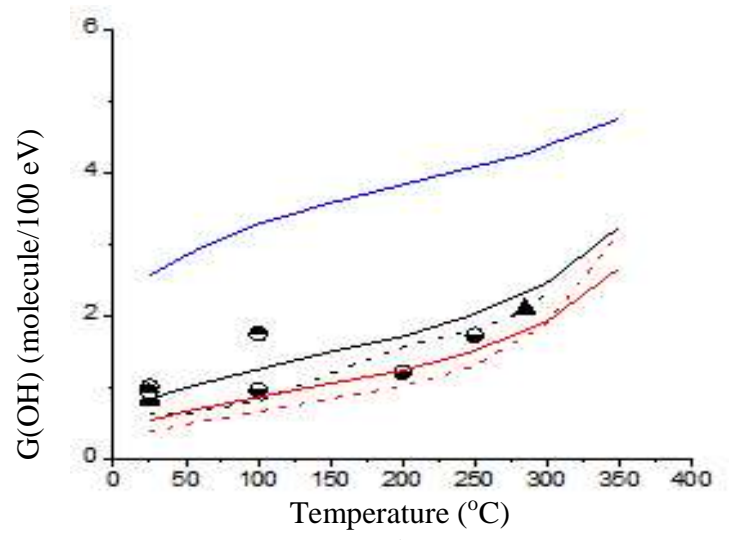

(b)

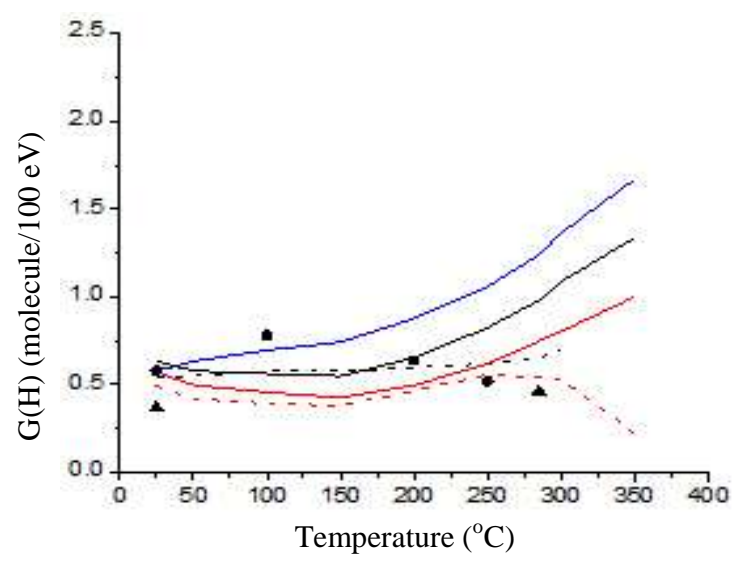

(c)

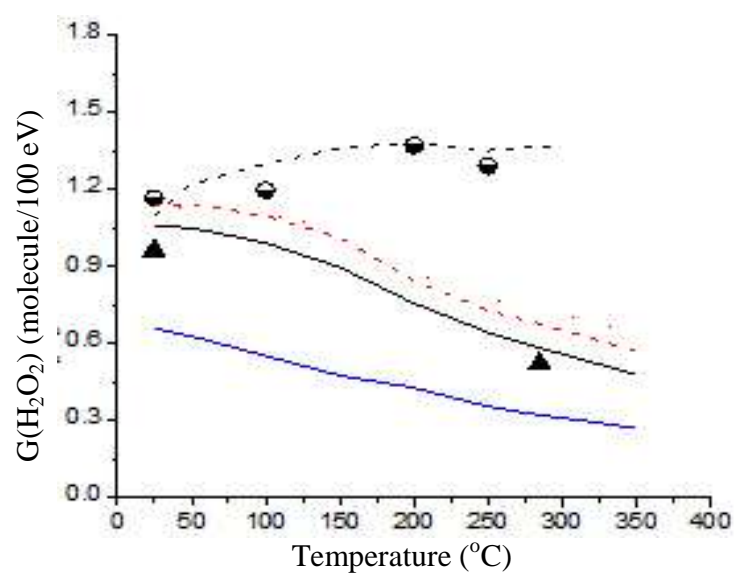

(d)

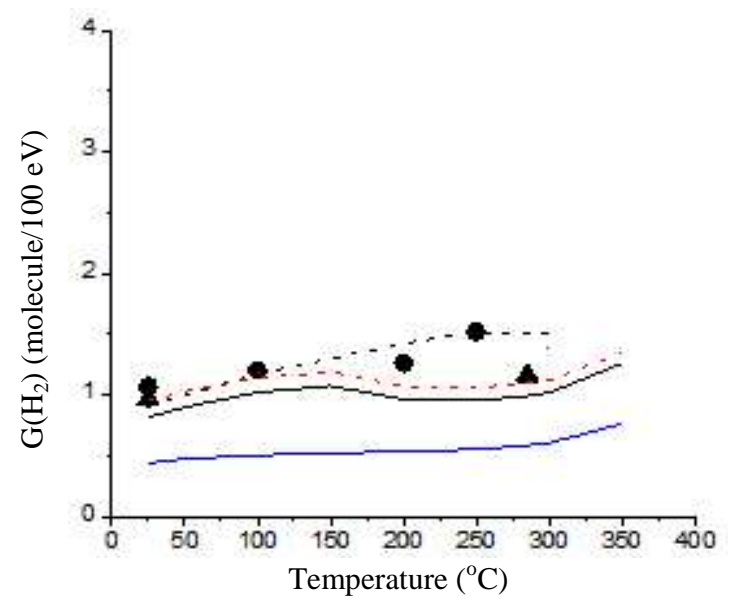

(e)

Fig. 1. Temperature dependence of the $G$-values (in molecules $/ 100 \mathrm{eV}$ ) for the liquid water radiolysis by $0.8-\mathrm{MeV}$ neutrons for the temperature range of $25-350^{\circ} \mathrm{C}$ : (a) $G\left(\mathrm{e}^{-}{ }_{\mathrm{aq}}\right)$, (b) $G\left({ }^{\circ} \mathrm{OH}\right),\left(\right.$ c) $G\left(\mathrm{H}^{*}\right),\left(\right.$ d) $G\left(\mathrm{H}_{2} \mathrm{O}_{2}\right)$, and (e) $G\left(\mathrm{H}_{2}\right)$. Our calculated results were obtained at $10^{-7}$ and $10^{-6} \mathrm{~s}$ based on the radiation effects of recoil protons whose energies are $0.5057,0.186$, and $0.0684 \mathrm{MeV}$; the graphs for $10^{-7}$ and $10^{-6} \mathrm{~s}$ are shown as solid and dashed red lines, respectively. Experimental data are from: [5] (•) (using YAYOI reactor as a source of fast-neutron at the University of Tokyo whose neutron energy is $\sim 0.8 \mathrm{MeV}$ ), [22] ( $\Delta$ ) (mean $G$-values from different research groups calculated by neglecting the highest and lowest values for each species). The solid black lines show the $2-\mathrm{MeV}$ fast neutron calculated $G$-values [15]. The dashed black lines are the results for 0.8 $\mathrm{MeV}$ fast neutrons before using the self-data base of [4]. The solid blue lines show the estimated primary (or "escape") yields for the low-LET $(\sim 0.3 \mathrm{keV} / \mu \mathrm{m})$ radiolysis of water [1,23].

For the sake of illustration, Fig. 2 shows the time evolution of various yields calculated from Monte Carlo simulations of the radiolysis of liquid water by $0.8-\mathrm{MeV}$ neutrons at two fixed temperatures, 25 and $350^{\circ} \mathrm{C}$. This figure gives information about the formation and decay of $\mathrm{e}^{-}$ and the yield of ${ }^{\circ} \mathrm{OH}$ at the range of time $\sim 10^{-12}-10^{-6}$ $\mathrm{s}$ after the deposition of ionizing radiation in water. It shows that all the rates of decay, rates of formation, and yields are higher at the higher temperature.

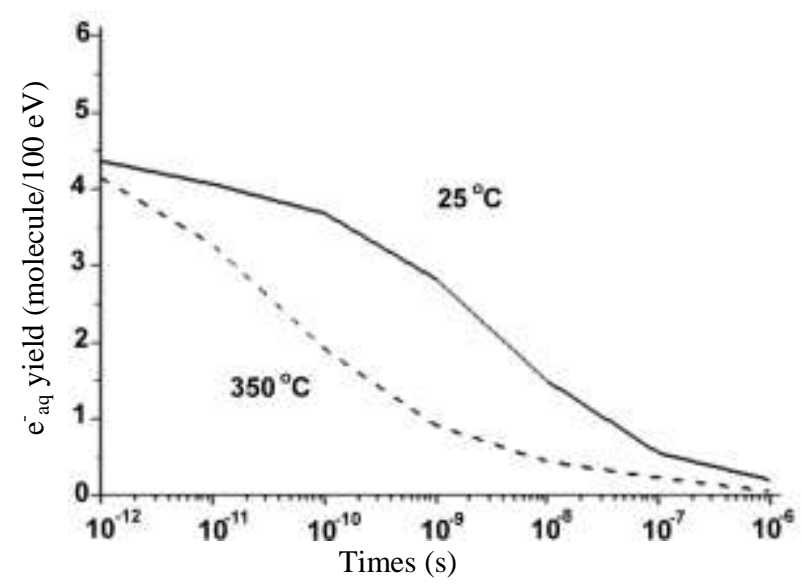

(a) 


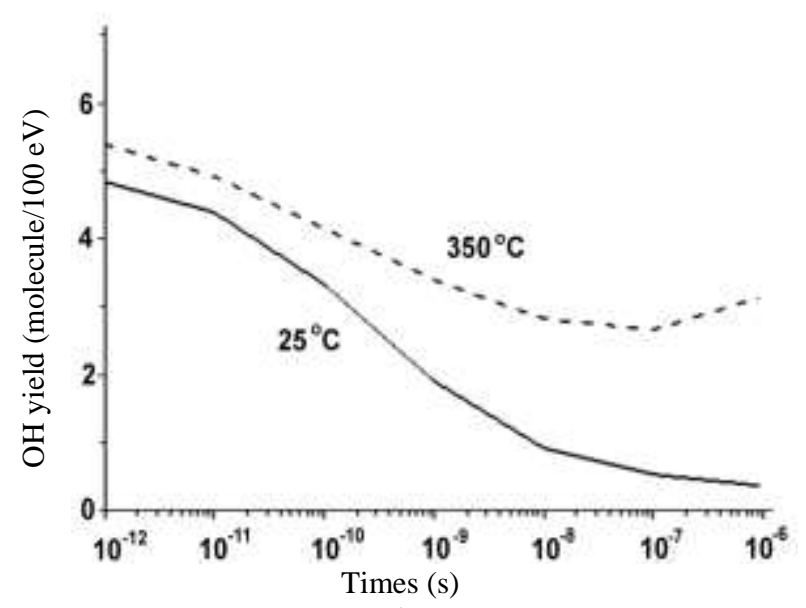

(b)

Fig. 2. Variation of the yields of $\mathrm{e}_{\text {aq }}^{-}$(a) and ${ }^{\circ} \mathrm{OH}$ (b) (in molecules $/ 100 \mathrm{eV}$ ) computed from Monte Carlo simulations of the radiolysis of liquid water by $0.8 \mathrm{MeV}$ fast neutrons as a function of time from $10^{-12}$ to $10^{-6} \mathrm{~s}$, for the two fixed temperature 25 and $350^{\circ} \mathrm{C}$ (solid and dashed lines, respectively).

\section{CONCLUSION}

Monte Carlo simulations were used to calculate the $G$-values for the primary species of the radiolysis of neutral liquid water by incident 0.8 $\mathrm{MeV}$ neutrons at the $25-350^{\circ} \mathrm{C}$ temperature range. The fast neutron $G$-values were predicted by calculating the yields of the first three recoil protons produced by the collision of neutrons with water by neglecting the radiation effects due to oxygen ion recoils. Overall, there is a reasonably good agreement between the simulated and existing experimental $G$-values for the entire temperature range. Our computed yields for $0.8-\mathrm{MeV}$ fast neutron radiation show essentially similar temperature dependences over the range of temperature studied with those for $2-\mathrm{MeV}$ fast neutron and low-LET radiation, but with lower values for yields of free radical yields and higher values for molecular yields. However, more experimental data are needed in order to compare our temperature dependence of primary species yields and to test the modeling calculations more thoroughly.

\section{REFERENCES}

1. S. Sanguanmith, Y. Muroya, J. Meesungnoen et al., Chem. Phys. Lett. 508 (2011) 224, and the references thereinafter.

\section{S. Le Caer, Water 3 (2011) 235.}

3. P.C. Burns, R.C. Ewry and A. Wavrotsky, Science 335 (2012) 1184.
4. D.M. Bartels, J. Henshaw and H.E. Sims, Radiat. Phys. Chem. 82 (2013) 16.

5. G.R. Sunaryo, Y. Katsumura and K. Ishigure, Radiat. Phys. Chem. 45 (1995) 703.

6. G.R. Sunaryo, J. Meesungnoen and J.-P JayGerin, Atom Indonesia 37 (2011) 29.

7. A.J. Elliot and D.M. Bartels, The Reaction Set, Rate Constants and $G$-values for the Simulation of the Radiolysis of Light Water Over the Range $20^{\circ} \mathrm{C}$ to $350^{\circ} \mathrm{C}$ Based on Information Available in 2008, Report AECL No.: 153127160-450-001, Atomic Energy of Canada Ltd., Ontario (2009).

8. L.M. Kohan, S. Sanguanmith, J. Meesungnoen et al., RSC Adv. 42 (2013) 19282.

9. L.M. Kohan, J. Meesungnoen, S. Sanguanmith et al., RSC Adv. 181 (2014) 495.

10. S. Mustaree, J. Meesungnoen, S.L. Butarbutar et al., RSC Adv. 4 (2014) 43572.

11. S.L. Butarbutar, Y. Muroya, L. Mirsaleh Kohan et al., Atom Indonesia J. 39 (2013) 51.

12. D. Swiatla-Wojcik, Chem. Phys. Let. 641 (2015) 51.

13. D. Swiatla-Wojcik and G.V. Buxton, Radiat. Phys. Chem. 79 (2010) 52.

14. C. Alcorn, J.C. Brodovitch, K. Ghandi et al., Chem. Phys. 435 (2014) 29.

15. S.L. Butarbutar, S. Sanguanmith, J. Meesungnoen et al., Radiat. Res. 181 (2014) 659.

16. S.L. Butarbutar, J. Meesungnoen, D.A. Guzonas et al., Radiat. Res. 182 (2014) 695.

17. V. Kanike, J. Meesungnoen and J.-P. Jay-Gerin, Austin J. Nucl. Med. Radiother. 2 (2015) 1011.

18. S. Sanguanmith, J. Meesungnoen and J.-P. JayGerin, Chem. Phys. Lett. 588 (2013) 82.

19. J. Meesungnoen, S. Sanguanmith and J.-P. JayGerin, RSC Adv. 5 (2015) 76813.

20. L.M. Kohan, J. Meesungnoen, S. Sanguanmith et al., Recent. Res. Devel. Physical. Chem. 11 (2014) 15.

21. S. Sanguanmith, J. Meesungnoen, D.A. Guzonas et al., Recent Res. Devel. Physical. Chem. 11 (2014) 1.

22. H. Christensen, Fundamental Aspects of Water Coolant Radiolysis, SKI Report 2066, Swedish Nuclear Power Inspectorat (2006).

23. S. Sanguanmith, J. Meesungnoen, Y. Muroya et al., Phys. Chem. Chem. Phys. 14 (2012) 16731. 